of life (range 11-18 days). Wilcoxon signed rank-test was used to test for differences in cTnT between the different timepoints.

Results Mean gestational age was 26.1 weeks (range 23.0-27.9) and mean birth weight $838 \mathrm{~g}(438-1287 \mathrm{~g})$. At postnatal day 3, median cTnT was $148 \mathrm{ng} / \mathrm{l}$ (range 82-386). cTnT decreased between day 3 and day 7 to $96 \mathrm{ng} / \mathrm{l}(68-214)$ ( $\mathrm{p}<0.001)$. Between one and two weeks of age, cTnT again increased to 144 ng/l (95-338) ( $\mathrm{p}=0.001)$. Thirty-four infants (57\%) were treated for a hemodynamically significant PDA (hsPDA) at a mean age of 8 days (SD 3.3). Twenty-three received only pharmacological treatment, 9 had surgery after pharmacological treatment and 2 had primary surgery. cTNT did not differ at any of the three time points between infants treated for hsPDA and infants not treated. Five infants who later died had significantly higher cTnT at 7 days of age than the 55 survivors (median $175 \mathrm{ng} / \mathrm{l}$, compared to $94 \mathrm{ng} / \mathrm{l})(\mathrm{p}=0.01$ ).

Conclusion cTnT levels in extremely preterm infants are tenfold higher than reference values in adults. We did not find any relation between cTnT and need for PDA-treatment in this study.

\section{PS-018 EVOLUTION OF SPECKLE TRACKING DERIVED 2-D STRAIN PARAMETERS IN VERY LOW BIRTH WEIGHT INFANTS WITH AND WITHOUT BRONCHOPULMONARY DYSPLASIA DURING THE NEONATAL PERIOD}

${ }^{1} \mathrm{C}$ Czernik, ${ }^{1} \mathrm{~S}$ Rhode, ${ }^{2} \mathrm{~S}$ Helfer, ${ }^{1} \mathrm{G}$ Schmalisch, ${ }^{1} \mathrm{C}$ Bührer, ${ }^{2} \mathrm{~L}$ Schmitz. ${ }^{1}$ Department of Neonatology, Charité University Berlin, Berlin, Germany; 'Department of Pediatirc Cardiology, Charité University Berlin, Berlin, Germany

\subsection{6/archdischild-2014-307384.314}

Background and aims In preterm infants, postnatal myocardial adaptation may be influenced by bronchopulmonary dysplasia (BPD). We aimed to describe the development of left ventricular function by serial 2D-Doppler, and speckle tracking echocardiography (2D-STE) in infants with and without BPD during the neonatal period in comparison to anthropometric and conventional haemodynamic parameters.

Methods Prospective echocardiography on day of life (DOL) 1, 7, 14, and 28 in 119 preterm infants $<1500 \mathrm{~g}$ birth weight, of whom 36 developed BPD (oxygen supplementation at 36 gestational weeks). Non-BPD and BPD infants differed significantly in median[IQR] gestational age (25.5[24-26.5] weeks vs. 29[2730 ]weeks, $\mathrm{p}<0.001)$ and birth weight (661[552-871]g vs. 1100 [890-1290]g, $\mathrm{p}<0.001)$.

Results The rapid growth of length and body weight during the first 4 weeks of life was not matched by increased speckle tracking parameters. Infants with BPD differed significantly $(p<$ 0.001) from those without BPD firstly, for all anthropometric parameters and conventional haemodynamic parameters except heart rate and secondly, for 2D-STE parameters global longitudinal systolic strain rate (GLSSR) and longitudinal systolic strain (LSSR) at the left free midwall segment. In infants with BPD, GLSSR $(\mathrm{p}<0.001)$ and LSSR $(\mathrm{p}<0.01)$ were significantly higher during the first week of life after which the differences disappeared. Low intra- and inter-observer variability was seen for longitudinal systolic strain and strain rate mid septum with a median coefficient of variation $<4.6 \%$.

Conclusions Reproducible 2D-STE measurements are possible in preterm infants $<1500 \mathrm{~g}$. There are early (DOL 1 and 7 ) ventricular changes (GLSSR and LSSR) in very low birth weight infants who develop BPD.

\section{PS-019 CHILDHOOD CARDIAC OUTCOME OF TWIN-TWIN TRANSFUSION SYNDROME AFTER INTRAUTERINE LASER TREATMENT: AN ECHOCARDIOGRAPHIC STUDY OF VENTRICULAR FUNCTION}

${ }^{1} \mathrm{C}$ Pegelow Halvorsen, ${ }^{2} \mathrm{LA}$ Mohlkert, ${ }^{2} \mathrm{M}$ Norman, ${ }^{3} \mathrm{SE}$ Sonesson. ${ }^{1} \mathrm{Clinical}$ Science and Education, Karolinska Institutet, Stockholm, Sweden; ${ }^{2}$ Clinical Science Intervention and Technology, Karolinska Institutet, Stockholm, Sweden; ${ }^{3}$ Women's and Children's Health, Karolinska Institutet, Stockholm, Sweden

\subsection{6/archdischild-2014-307384.315}

Background and aim Intrauterine laser treatment of placental vessels is used to alleviate abnormal circulatory load and cardiac morbidity affecting fetuses with twin-twin transfusion syndrome (TTTS). As the final cardiac outcome after such intervention is not known, we conducted this childhood follow-up study of cardiac function in twins treated with intrauterine laser for TTTS.

Methods and results Nineteen TTTS pairs (11 male, 8 female) with a mean age of 4.5 (range 1.1-9.9) years were assessed with echocardiography 2D, Doppler, DTI, 3D and speckle tracking, and compared with nineteen age- and gender-matched singletons.

Recipients had a lower left ventricular E/A ratio compared with their donor twins [1.48(0.35) vs. 1.66(0.28), $\mathrm{p}<0.05]$ but not compared with singleton controls, and all but one observation were within normal reference limits. There was no significant difference in the right ventricular E/A ratio $[1.26(0.28)$ vs. 1.41(0.28), $\mathrm{p}=0.06$ ], but recipients showed a slightly lower right ventricular e'/a' ratio when DTI was used. TTTS twins had smaller longitudinal left and right ventricular dimensions than controls. Besides a marginally lower ventricular strain in donors compared with controls, speckle tracking could not demonstrate any group differences in systolic ventricular function.

Conclusion Despite significantly different fetal cardiac loading conditions, survivors of laser-treated TTTS show only minor within-pair differences in diastolic cardiac function at follow-up. Cardiac function in TTTS twins compare well to singleton controls, suggesting a favourable long-term outcome.

\section{PS-020 CARDIAC FUNCTION IN NEWBORNS WITH FETAL GROWTH RESTRICTION: MORPHOLOGICAL AND FUNCTIONAL CHANGES}

${ }^{1} \underline{L}$ Rodriguez Guerineau, ${ }^{2} \mathrm{M}$ Perez Cruz, ${ }^{1} \mathrm{FJ}$ Cambra, ${ }^{2} \mathrm{O}$ Gómez, ${ }^{3}$ J Carretero, ${ }^{2} \mathrm{MD}$ Gomez Roig, ${ }^{2} \mathrm{~F}$ Crispi, ${ }^{3} \mathrm{~J}$ Bartrons. ${ }^{1}$ Pediatric Intensive Care Unit, Hospital Sant Joan de Déu, Esplugues de Llobregat, Spain; ${ }^{2}$ Department of Obstetrics and Gynecology, Barcelona Center for Maternal Fetal and Neonatal Medicine Hospital Clínic and Hospital Sant Joan de Déu, Barcelona, Spain; ${ }^{3}$ Pediatric Cardiology, Hospital Sant Joan de Déu, Esplugues de Llobregat, Spain

10.1136/archdischild-2014-307384.316

\begin{tabular}{|c|c|c|c|}
\hline Characteristics $(n=50)$ & $\mathrm{FGR}^{\#}$ & Controls $^{\#}$ & $\mathrm{p}$ \\
\hline Gestational age at birth & $37 w+6 d$ & $39 w+4 d$ & \\
\hline$(w=$ weeks $+d=$ days $)$ & $(34 w-40 w)$ & $(34 w-41 w)$ & 0,001 \\
\hline Weight & 2,23 & 3,24 & \\
\hline \multirow[t]{2}{*}{$(\mathrm{kg})$} & $(1,58-2,73)$ & $(1,99-4,00)$ & $<0,001$ \\
\hline & 1,56 & 1,90 & \\
\hline \multirow[t]{2}{*}{ Right sphericity index } & $(1,11-2,28)$ & $(1,53-2,46)$ & $<0,001$ \\
\hline & 1,63 & 1,98 & \\
\hline Left sphericity index & $(1,32-2,32)$ & $(1,61-2,63)$ & $<0,001$ \\
\hline
\end{tabular}


Background and aims The adaptive changes of the fetal heart in fetal growth restriction (FGR) could persist into childhood and be responsible for the increased cardiovascular mortality rate in adulthood. The aim of the study was to assess cardiac morphology and function in newborns with FGR.

Methods FGR was defined as a birth weight centile $\leq 10$. Prospective study of 50 neonates, 25 with FGR and 25 with normal intrauterine growth and weight at birth (Table 1). Comprehensive echocardiographic study was performed assessing cardiac morphology, systolic and diastolic function.

Results Compared with controls, neonates with FGR had more globular cardiac ventricles (Table 1), lower systolic excursions of the tricuspid and mitral valvular plane and lower values of the s' in the lateral and septal mitral annulus in the tissue Doppler imaging (TDI) study $(\mathrm{p}<0,05)$. The e' at the tricuspid, lateral and septal mitral annulus together with the $\mathrm{E}$ wave of tricuspid inflow were significantly reduced in the FGR group; and tricuspid deceleration time showed a trend to increase without reaching statistical significance.

Conclusions Newborns with FGR manifest cardiac shape changes, reduced systolic values of the TDI at the left heart and lower values of diastolic function more pronounced at the right heart compared with neonates with normal intrauterine growth.

\section{PS-021 ELECTRICAL CARDIOMETRY STROKE VOLUME EVALUATION IN NICU: COMPARISON WITH FUNCTIONAL ECHOCARDIOGRAPHY}

${ }^{1} \mathrm{~A}$ Boet, ${ }^{2} \mathrm{G}$ Jourdain, ${ }^{3} \mathrm{~A}$ Capderou, ${ }^{4} \mathrm{O}$ Grollmuss, ${ }^{1} \mathrm{P}$ Labrune, ${ }^{1} \mathrm{D}$ De Luca, ${ }^{4} \mathrm{~S}$ Demontoux. ${ }^{1}$ Pediatrics and Neonatal Critical Care, CHU Antoine Béclère South Paris University Hospital, Clamart, France; ${ }^{2}$ Pediatrics and Neonatal Critical Care Pediatric S. M. U. R, CHU Antoine Béclère South Paris University Hospital, Clamart, France; ${ }^{3}$ INSERM 999, South Paris University Hospital, Le Plessis Robinson, France; ${ }^{4}$ Centre Chirurgical Marie Lannelongue, South Paris University Hospital, Le Plessis Robinson, France

\subsection{6/archdischild-2014-307384.317}

Background Evaluation of cardiac output in neonates might be difficult because of the complexity and risks of invasive classical procedures. New systems like electrical cardiometry (EC: Osypka Medical, Berlin, Germany and La Jolla, California, USA) have been proposed but few data are available in neonates. We investigated stroke volume (SV) using EC in term and preterm infants.

Methods Eligible patients were neonates admitted to the NICU and undergoing echocardiography for any clinical reasons, without congenital heart disease. We measured SV with EC and echocardiography, within $10 \mathrm{~min}$. Measurements were repeated 6 times by the same operator to calculate repeatability before and after echocardiography. Data have been compared with correlation and Bland-Altman analysis.

Results 59 neonates were enrolled, allowing 150 paired measurements. Mean gestational age and birth weight were $33.9 \pm$ 3.4 wks and $1988 \pm 823$ g, respectively.

Results of Pearson correlation and Bland-Altman analysis for the whole population were $(\mathrm{r}=0.611 ; \mathrm{p}<0.001)$ and (mean error [echo-EC] $-1.35 \mathrm{~mL}$ [95\% CI: $-6.55 \mathrm{~mL} \pm 3.85 \mathrm{ml}])$, respectively.

Correlation is maintained even with PDA ( $\mathrm{r}=0.627$; $\mathrm{p}<$ 0.001).

Gestational age seems to do not influence the correlation between EC and echo (Partial correlation coefficient $r=0.36$; $p$ $<0.0001)$.

Repeatability (coefficient of variation) was 46\% for EC and $52 \%$ for echocardiography. There was no difference in SV measured by EC after $10 \min (3.76 \pm \mathrm{SD}$ vs $3.78 \pm \mathrm{SD} ; \mathrm{p}=$ 0.56 , Wilcoxon test).

Conclusions EC is feasible, reproducible and quick. It could be an useful tool for continuous monitoring and haemodynamic evaluation in neonates. EC is particularly interesting for the clinical management of preterm neonates.

\section{PS-022 WITHDRAWN}

\section{Congenital Heart Disease}

\section{PS-023 THE PREVALENCE AND SPONTANEOUS CLOSURE RATE OF ISOLATED VENTRICULAR SEPTAL DEFECT IN NEWBORNS BY ECHOCARDIOGRAPHIC SCREENING}

E Feil. Paediatric Cardiology, Marien Hospital Darmstadt, Darmstadt, Germany

\subsection{6/archdischild-2014-307384.318}

Aims Evaluation of the prevalence and spontaneous closure rate of the most common congenital heart defect (CHD) - the ventricular septal defect (VSD) - in one maternity clinic, using colour flow Doppler echocardiographic screening (ECHO).

Methods Over a period of 7 years ECHO was offered to all babies who were born at the Marien hospital in Darmstadt. An experienced paediatric cardiologist performed the ECHO using a $10 \mathrm{MHz}$ transducer within the first $72 \mathrm{~h}$ of neonatal life. The prevalence of different types of VSD and their outcome were evaluated.

Results 8082 neonates were screened. 399 cases (49/1000) of CHD were detected by ECHO. VSD was found in 320 neonates (40/1000) (137 male, 183 female): 4 perimembranous, 24 multiple and 292 muscular VSD. 2 major, 16 hemodynamically significant and 304 minor VSD. 46 had a typical murmur (14\%), 274 were without clinical sign (including 1 major VSD). In the follow-up ( 3 month to 6,7 years) 280 could be included: the 2 major VSD had to be closed interventionally and surgically within the first year of life. The spontaneous closure rate was $89 \%$ after 0,2-4,5 years (average 0,6) (hemodynamically significant: 50\%, minor VSD: 93\%). All VSD without spontaneous closure after 1 year (22 cases) had a typical murmur.

Conclusions The prevalence of VSD is considerably high in neonates when ECHO is performed. There are slightly more female neonates with this diagnosis. The spontaneous closure rate is high regarding minor VSD. Auscultation is insufficient to diagnose VSD in neonatal period but is excellent in the follow-up to detect VSD without spontaneous closure.

\section{PS-024 NEURODEVELOPMENTAL EXAMINATION BY BAYLEY SCALE OF INFANT DEVELOPMENT-II IN CHILDREN WITH CYANOTIC CONGENITAL HEART DISEASE}

ZI Yilmaz, F Genel, E Ozbek, B Erdur, T Mese, U Karaaslan. Department of Pediatrics, Dr. Behcet Uz Children's Hospital, Izmir, Turkey

10.1136/archdischild-2014-307384.319

Background and aims The aim of the study was to make the neurodevelopmental evaluation of the children with cyanotic congenital heart disease.

Methods Children between the age of six to fourty-two months were included in the study and were evaluated in three groups 